\title{
Perfiles momentáneos de compromiso a lo largo de tres clases de ciencias en estudiantes colombianos
}

\section{Momentary Profiles of Engagement Throughout Three Classes of Sciences in Colombian Students}

\author{
Solanlly Ochoa-Angrino ${ }^{1}$, Jairo Andrés Montes-Gonzáles², Tatiana Rojas-Ospina ${ }^{3}$ \\ y Marcela Valencia-Serrano ${ }^{4}$ \\ ${ }^{1}$ Psicóloga. Magíster en Psicología. Doctora en Educación, Mención en Psicología Educativa. \\ Profesora del Departamento de Ciencias Sociales, Pontificia Universidad Javeriana de Cali. \\ E-mail: sochoa@javerianacali.edu.co
}

${ }^{2}$ Psicólogo. Magíster en Psicología. Doctor en Psicología. Profesor del Departamento de Ciencias Sociales, Pontificia Universidad Javeriana de Cali. E-mail: jairomontes@gmail.com

${ }^{3}$ Psicóloga. Magíster en Psicología. Doctora en Psicología. Profesora del Departamento de Ciencias Sociales, Pontificia Universidad Javeriana de Cali. E-mail: ctrojas@javerianacali.edu.co

${ }^{4}$ Psicóloga, Magíster en Educación, Estudiante del Doctorado en Psicología. Profesora del Departamento de Ciencias Sociales, Pontificia Universidad Javeriana de Cali.

E-mail: marcelav@javerianacali.edu.co

Este artículo es producto del proyecto de investigación "Enseñanza de las ciencias en educación media (Fase I). Interacciones entre profesores y estudiantes en el salón de clase durante la enseñanza de ciencias" (Código 4425), financiado por la Pontificia Universidad Javeriana Cali. Oficina de Investigación, Desarrollo e Innovación.

Los autores agradecen a la Prof. Lucero Aragón Espinosa, de la Pontificia Universidad Javeriana Cali, por la revisión de este artículo.

Pontificia Universidad Javeriana Cali.

Santiago de Cali, Colombia.

\section{Resumen}

A partir de una aproximación orientada a la persona (Conner y Pope, 2013; Schmidt, Rosenberg y Beymer, 2017), este estudio examina perfiles momentáneos de compromiso cognitivo, afectivo y comportamental en estudiantes de secundaria a lo largo de tres sesiones de clases de ciencias. Participaron 53 estudiantes de secundaria colombianos (63 \% mujeres, de $9 .^{\circ}$ a $11 .^{\circ}$ grado). El método de muestreo de experiencias (Csikszentmihalyi y Larson, 1987; Zuzanek, 1999) se usó para tomar medidas repetidas del compromiso cognitivo (importancia), afectivo (disfrute e interés) y comportamental (concentración y esfuerzo). Se realizó un análisis de agrupamiento o clustering para la identificación de los perfiles. El análisis de agrupamiento permitió identificar cinco perfiles momentáneos de compromiso que se distribuyen en tres tipos por cada sesión: moderado, universal- 
mente bajo, renuente, racional y recreacional. En la primera sesión los estudiantes se distribuyen de la siguiente manera: moderado (43 $\%$ ), recreacional (32\%) y universalmente bajo (25\%); en la segunda sesión, renuente (44\%), moderado (38\%), y racional $(18 \%)$; y, en la tercera sesión, moderado (47\%), racional (32 $\%$ ) y universalmente bajo (21\%). Los perfiles mostraron permanencia y variación. El perfil más frecuente y permanente fue el compromiso moderado, mientras que el más variable fue el universalmente bajo. Se concluye que los perfiles momentáneos de compromiso plantean preguntas y retos diferenciados para los educadores, quienes deben desarrollar prácticas educativas que promuevan perfiles de compromiso relacionados con mejores procesos de aprendizaje y desempeño en ciencias. Los resultados se discuten en términos de las implicaciones educativas en las interacciones profesor-estudiante.

Palabras clave: compromiso académico; compromiso en ciencias; perfiles de compromiso; análisis orientado a la persona; método de muestreo de experiencias.

\section{Abstract}

Based on a person-oriented approach (Conner y Pope, 2013; Schmidt, Rosenberg y Beymer, 2017), this study examines momentary profiles of cognitive, affective, and behavioral engagement in high school students through three sessions of science classes (e.g. environmental education, biology, chemistry, and physics). A total of 53 Colombian high school students participated in this study (63 $\%$ women, 9 th to 11 th grade). The experience sampling method (ESM; Csikszentmihalyi y Larson, 1987; Zuzanek, 1999) was used to take repeated measures of cognitive engagement (importance), affective engagement (enjoyment and interest) and behavioral engagement (concentration and effort). Through a cellphone alarm, the students were called to answer the Experience Sampling Form (ESF) once in each class throughout the thematic unit studied. In the ESF students reported their thoughts, emotions and efforts at the moment of the signal. A total of 159 students' responses were collected. A clustering analysis was performed to identify the profiles. The cluster analysis allowed the identification of five momentary engagement profiles that were classified into three types for each session: moderate, universally low, reluctant, rational, and recreational. In the first session, the students were distributed as follows: Moderate (43\%), Recreational (32\%) and Universally Low (25\%); in the second session, Reluctant (44\%), Moderate (38\%), and Rational (18\%); and in the third session, Moderate (47\%), Rational (32\%) and Universally Low (21 \%). That profiles showed permanence and variation. The most frequent and permanent engagement profile was the moderate, while the most variable was the universally low. When reviewing the profiles by session, we found that the groups that make up these profiles were not the same students. This not only marks a variability in the profiles between sessions, but also allows the description of the oscillations and intraindividual trajectories of engagement. This implies that the same student does not necessarily present the same pattern of engagement throughout the three classes sessions and, although some students tend to have stable profiles over time, others show greater variability. It is concluded that momentary profiles of academic engagement pose different questions and challenges for educators, in terms of educational practices that may promote engagement profiles linked to deep learning processes and performance in sciences. Results are discussed in terms of the educational implications in teacher-student interactions. Future research should combine three elements in the study of engagement: to include multidimensionality of engagement (e.g., cognitive, affective and behavioral), to describe momentary profiles, and to establish relationship among these profiles with the characteristics of educational environments 
(e.g., educational practices and relationships between teachers and students).

Keywords: academic engagement; science engagement; engagement profiles; personoriented analysis; experience sampling method.

\section{Introducción}

Un estudiante comprometido con su formación académica se caracteriza por su responsabilidad con las metas educativas, el interés en ellas, el disfrute cuando las realiza y una energía que lo impulsa a estudiar por aprender, por darle sentido al mundo y enfrentar los retos de su vida cotidiana. Los estudiosos del compromiso señalan que es un constructo multidimensional que incluye factores cognitivos, afectivos y comportamentales (Christenson, Reschly y Wylie, 2012; Conner y Pope, 2013; Lam et al., 2016; Schmidt, Rosenberg y Beymer, 2017). El compromiso cognitivo se refiere al grado en que los estudiantes perciben que las actividades académicas son valiosas para ellos y para la sociedad en la que viven (grado de relevancia personal y social); el compromiso afectivo implica sentimientos positivos como el interés, el entusiasmo y el disfrute de los estudiantes hacia las actividades de aprendizaje y la escuela; el compromiso comportamental se refiere a la participación, el esfuerzo puesto en las tareas académicas, la energía que ponen los estudiantes en su realización, y la persistencia y constancia para conseguir y mantener adecuados procesos de aprendizaje y logros en las tareas académicas.

El compromiso se ha vuelto un tema de particular importancia para el campo educativo porque hay suficiente evidencia que muestra su relación con los procesos de permanencia en la escuela, el aprovechamiento de oportunidades de aprendizaje y el logro de metas académicas. Furrer, Skinner y Pitzer (2014) reportan relaciones significativas positivas entre compromiso y bienestar psicológico representado en alta autoestima, responsabilidad, buenas relaciones sociales y esperanza. Por el contrario, la falta de compromiso académico se ha asociado con ausentismo escolar, resultados académicos pobres, pérdida de cursos, deserción y con problemas de depresión y agresión (Jessor y Jessor, 1977, citado por Shernoff y Bempechact, 2014).

A pesar del gran número de investigaciones sobre el compromiso en la última década, diversos autores señalan que hay serios problemas en su estudio; uno de los más importantes es la manera en la que se analiza (Conner y Pope, 2013; Schmidt et al., 2017). Para describir este problema, estos autores señalan que hay dos tipos de abordajes: un abordaje orientado a la variable y un abordaje orientado a la persona.

Las investigaciones que realizan un análisis orientado a la variable estudian el compromiso como una medida agregada de las tres dimensiones creando promedios (por ejemplo, Shernoff, Ruzek y Sinha, 2016). En estos estudios se reporta un nivel de compromiso general que no establece diferencias cualitativas entre las dimensiones.

Schmidt et al. (2017) señalan que los diferentes tipos de compromiso afectan también de manera diferente el desempeño y el esfuerzo de los estudiantes. Es por ello que se propone un análisis orientado a la persona en el cual se identifiquen perfiles que representen las tres dimensiones del compromiso en actividades académicas particulares.

Los estudios recientes basados en el análisis orientado a la persona plantean que el concepto de "compromiso" tiene una naturaleza emergente y multifacética, que en muchos casos dificulta su medición y el planteamiento de una teoría unificada (Eccles, 2016). Desde esta perspectiva se responde a los recientes llamados a la psicología a centrarse en el carácter ideográfico de los fenómenos complejos (Martin et al., 2015; Molenaar, 2004; Salvatore y Valsiner, 2010; Van Geert y Van Dijk, 2002). No obstante, estas aproximaciones tienen en cuenta aspectos como las trayectorias intraindividuales, y las dinámicas de compromiso y motivación en tiempo real, 
aspectos considerados de poca importancia por algunos investigadores en disciplinas aplicadas como la psicología educativa (Martin, Papworth, Ginns, Malmberg, Collie y Calvo, 2015).

Dentro de esta literatura emergente se destacan dos vertientes de trabajos. Un primer tipo de investigaciones desde la perspectiva orientada a la persona se basan en la caracterización de trayectorias longitudinales del compromiso, enfocadas en tópicos como: la relación entre trayectorias de compromiso y la desconexión académica de estudiantes de secundaria (Jang, Kim y Reeve, 2016), las trayectorias de compromiso emocional y burnout escolar en estudiantes de secundaria y su relación con su desempeño académico y síntomas depresivos, y cómo los niños de últimos años de escuela primaria desarrollan su compromiso comportamental en función de sus relaciones con los pares y los profesores (De Laet et al., 2015). Estos estudios longitudinales, aunque examinan la variabilidad intra e interindividual a lo largo de amplios periodos de tiempo (meses y años) y desagregan los elementos del compromiso, generalmente no capturan los cambios que van ocurriendo en escalas de tiempo más pequeñas (Siegler, 1994).

El segundo tipo de investigaciones de esta perspectiva orientada a la persona parte de la caracterización de perfiles. En esta categoría se pueden citar trabajos sobre diferencias individuales en compromiso y éxito académico en estudiantes universitarios (Ketonen et al., 2016), estabilidad y cambio en los perfiles de compromiso en estudiantes universitarios (Gillet, Morin y Reeve, 2017), análisis de perfiles de disposición al compromiso en estudiantes de secundaria (Lawson y Masyn, 2015), perfiles de compromiso y su relación con el burnout en estudiantes de secundaria (Salmela-Aro, Moeller, Schneider, Spicer y Lavonen, 2016), motivación y compromiso en tiempo real en estudiantes de secundaria (Martín et al., 2015), y perfiles de compromiso en estudiantes de matemática de secun- daria (Watt, Carmichael y Callingham, 2017).

Utilizando una aproximación orientada a la persona, Conner y Pope (2013) examinaron los perfiles de compromiso escolar en niños que asistían a colegios de alto rendimiento. Participaron 6294 estudiantes de 15 colegios. Primero diferenciaron los tres tipos de compromiso, definiéndolos como se presenta a continuación.

Cognitivo: los estudiantes perciben las actividades académicas como valiosas para ellos y para la sociedad.

Afectivo: Los estudiantes experimentan sentimientos positivos como interés, entusiasmo y disfrute hacia la escuela y hacia las actividades de aprendizaje.

Comportamental: participación, esfuerzo, energía, persistencia y constancia que los estudiantes ponen en las tareas académicas para conseguir y mantener adecuados procesos de aprendizaje.

Luego analizaron los patrones que reportaban los estudiantes y a partir de ellos, identificaron siete perfiles de compromiso: (1) intencional, que implica alto compromiso comportamental y cognitivo, pero bajo compromiso afectivo; (2) total, que implica alto compromiso afectivo, comportamental y cognitivo; (3) racional, alto compromiso cognitivo y bajo compromiso afectivo $\mathrm{y}$ comportamental; (4) renuente, alto compromiso comportamental, pero bajo compromiso emocional y cognitivo; (5) agradable, alto compromiso afectivo, pero bajo compromiso comportamental y cognitivo; (6) mentalizado, alto compromiso cognitivo y afectivo, pero bajo compromiso comportamental; y (7) recreacional, alto compromiso afectivo y comportamental y bajo compromiso cognitivo. El estudio reporta que, aunque el $84 \%$ de los participantes indicó que se esforzaban mucho (compromiso renuente), menos de la tercera parte mostraba un compromiso total; es decir, altos niveles de compromiso cognitivo, afectivo y comportamental.

Aunque Schmidt et al. (2017) reconocen los aportes de Conner y Pope (2013) y retoman 
sus categorías para estudiar el compromiso, señalan como limitación que estos autores estudian el compromiso de manera transversal, como un fenómeno estable; no indagan por la variación del compromiso a lo largo del tiempo, en diferentes momentos de aprendizaje-enseñanza. Basados en el estudio de Conner y Pope (2013), pero estudiando patrones de compromiso en el momento de participación de los estudiantes en actividades académicas, Schmidt et al. (2017) identificaron seis patrones de compromiso momentáneo en estudiantes de secundaria. Los autores señalan que los estudiantes, la mayoría del tiempo, mostraron altos niveles de compromiso de un tipo (por ejemplo, comportamental), pero bajo en otros. Los estudiantes exhibieron bajos niveles de compromiso en las tres dimensiones en alrededor del $22 \%$ del tiempo en que fueron observados; el compromiso total fue el menos frecuente, reportado únicamente el $11 \%$ del tiempo.

El presente estudio está en línea con investigaciones recientes sobre compromiso que señalan la necesidad de estudiarlo como un fenómeno multidimensional (cognitivo, afectivo y comportamental) (Christerton et al., 2012; Conner y Pope, 2013; Lam et al., 2016; Schmidt et al., 2017). Al igual que Conner y Pope (2013) y Schmidt et al. (2017), estudia el compromiso en términos de perfiles y avanza en relación con Schmidt et al. (2017) en la medida en que toma en cuenta la dimensión temporal al mostrar los perfiles de compromiso a lo largo de una unidad temática. Estudios de este tipo no se encuentran en Latinoamérica pues, aunque se empiezan a estudiar experiencias óptimas como el compromiso y la felicidad en contextos educativos (Hernández y Carranza, 2017; Rigo, 2017), aún no se avanza hacia la descripción de sus patrones.

Este estudio también hace énfasis en el compromiso en clases de ciencias, dada la relevancia del tema para el contexto colombiano. Los resultados de las Pruebas Saber en los diferentes niveles escolares $\left(5 .^{\circ}, 9 .^{\circ}\right.$ y $\left.11 .^{\circ}\right)$ suelen mostrar que los estudiantes obtienen niveles mínimos o medios de rendimiento en la mayoría de las áreas, siendo ciencias naturales una de las más críticas. Este patrón se evidencia también en pruebas internacionales como el Programme for International Student Assesment (PISA), en la que los estudiantes colombianos suelen ocupar los últimos lugares de rendimiento en áreas como lectura, matemática y ciencia (Ministerio de Educación Nacional de Colombia, 2017).

Estas dificultades en el desempeño de los estudiantes están asociadas con factores motivacionales, como el compromiso académico hacia las asignaturas y los patrones de interacción profesor-estudiante. Desde la psicología educativa, autores como Eccles (2016), Wang y Eccles (2013) y Wang y Holcombe (2010) plantean que los estudiantes con niveles de compromiso académico bajos en sus tres dimensiones (cognitivo, comportamental y afectivo) suelen aburrirse y perder interés en las asignaturas, además de encontrar poco valor en los contenidos y adoptar una actitud pasiva en las clases, lo cual puede afectar negativamente su desempeño. Este planteamiento ha sido soportado por diversos metaanálisis que concluyen que el compromiso académico en sus dimensiones cognitiva, comportamental y emocional presenta relaciones causales moderadas y fuertes con el rendimiento de los estudiantes, independientemente del área de conocimiento y del grado escolar (Chang, Chien y Chou, 2016; Lei, Chui y Zhou, 2018). Por el contrario, estudiantes que percibían sus tareas académicas como relevantes, que sentían que los contenidos y tareas se ligaban con sus intereses personales, presentaron un alto compromiso académico y buen rendimiento (Wang y Eccles, 2013). Estos estudios concluyen que la importancia percibida de las tareas influye en el compromiso y el desempeño.

En síntesis, este estudio contribuye a la investigación sobre el compromiso cognitivo, afectivo y comportamental de los estudiantes hispanos utilizando medidas de compromiso situacional a partir del muestreo de experiencias. Igualmente, adoptando una perspectiva 
orientada a la persona, describe subgrupos de estudiantes con diversos patrones de compromiso a lo largo de una unidad temática de ciencias, señalando su permanencia o cambio en determinados perfiles de compromiso.

Las preguntas de investigación que guían este estudio son: (1) ¿qué tipo de perfiles momentáneos caracterizan el compromiso de los estudiantes en las clases de ciencias?; (2) ¿los perfiles de compromiso de los estudiantes varían o se mantienen a lo largo de la unidad temática?

De acuerdo con la revisión de la literatura y las preguntas de investigación, este estudio evalúa dos hipótesis: (1) la variación momentánea del compromiso cognitivo, afectivo $\mathrm{y}$ comportamental origina diferentes perfiles de compromiso en los estudiantes durante las clases de ciencias. Esta hipótesis está basada principalmente en los estudios de Conner y Pope (2013) y Schmidt et al. (2017). Y (2) los perfiles de compromiso de los estudiantes varían en las clases de ciencias a lo largo de una unidad temática. Esta hipótesis se basa en el estudio de Martín et al. (2015), quienes encontraron variación diaria, semanal y mensual en el compromiso de estudiantes de secundaria.

\section{Método}

\section{Tipo de estudio}

El estudio que se presenta es de tipo descriptivo con medidas repetidas y análisis basados en clústeres.

\section{Participantes}

A partir de un muestreo intencional no probabilístico, se seleccionaron 53 estudiantes colombianos de secundaria de tres instituciones educativas, dos públicas y una privada, de los municipios de Palmira y Cali (Valle del Cauca, Colombia). La edad promedio de los participantes fue de 15 años $(M=15, D E=1.77), 63$ $\%$ mujeres; $40 \%$ de $9 .^{\circ}$ grado, $47 \%$ de $10 .^{\circ} \mathrm{y}$ $13 \%$ de $11 .{ }^{\circ}$. Las medidas se tomaron en los siguientes cursos: Biología (9. ${ }^{\circ}$ ) $40 \%$, Química $\left(10 .^{\circ}\right) 47 \%$ y Física $\left(11 .^{\circ}\right), 13 \%$. Los estudiantes participaron voluntariamente, previo consentimiento y asentimiento informado.

\section{Instrumentos}

El Cuestionario de Motivación Emergente (CME) usado en esta investigación es una adaptación del instrumento usado por Schmidt y Smith (2008) y validado por Ochoa-Angrino, Montes-Gonzáles y Rojas-Ospina (2018) para la población colombiana. El cuestionario está compuesto por 24 ítems que indagan por los estados emocionales y cognitivos de los estudiantes durante la realización de actividades académicas en el salón de clases; se califica con una escala tipo Likert, rango de 1 a 5 , donde 1 significa "nada" y 5 significa "mucho". Las medidas de las diferentes dimensiones del compromiso fueron tomadas de los promedios de ítems del CME.

Compromiso cognitivo: promedio de las respuestas de los estudiantes a los ítems: “Esta actividad era importante para ti?” $\mathrm{y}$ "¿Esta actividad era importante para tus metas futuras?" $(\alpha=.77)$.

Compromiso afectivo: promedio de las respuestas de los estudiantes a los ítems “Disfrutaste lo que estabas haciendo?” y “Esta actividad fue interesante para ti?” $(\alpha=.89)$.

\section{Compromiso comportamental:}

promedio de los ítems "¿Qué tan concentrado estabas?" y "¿Qué tanto te estabas esforzando?" $(\alpha=.61)$.

La confiabilidad de los ítems se considera adecuada y está dentro de los rangos reportados por otros estudios en compromiso que usan el CME (Salmela-Aro et al., 2016; Schmidt et al., 2017).

\section{Procedimiento}

La experiencia subjetiva de los estudiantes sobre el compromiso cognitivo, afectivo y comportamental durante las clases de ciencias 
se evaluó utilizando el método de muestreo de experiencias (MME) (Csikszentmihalyi y Larson, 1987; Zuzanek, 1999). Este método se utiliza para tomar muestras de los reportes de las experiencias subjetivas de las personas (cognitivas y emocionales) cuando participan en diferentes actividades cotidianas, entre ellas, las actividades propias de los contextos educativos. Hektner, Schmidt y Csikszentmihalyi (2007) reportan alta validez ecológica del MME, pues tomar las percepciones de las personas sobre tareas que realizan en el momento en que las están realizando reduce fallos atribuibles a la memoria y a la posibilidad de respuestas socialmente deseables.

Previo a la recolección de datos, y de acuerdo con el protocolo de uso del MME, los investigadores explicaron la manera de diligenciar el CME a los estudiantes que aceptaron participar voluntariamente en la investigación. Esta decisión fue apoyada por los padres de cada uno de los niños, quienes autorizaron su participación en el estudio mediante la firma del consentimiento informado. Durante la recolección de datos, los estudiantes fueron llamados, a través de una alarma de celular, a responder una (1) vez durante cada clase al CME a lo largo de la unidad temática estudiada. Para evitar disrupciones y tomar registro de una mayor variedad de actividades en las clases, los participantes de cada grupo fueron divididos a la mitad y cada subgrupo respondió en momentos diferentes de la clase. Al final de la recolección de datos, cada estudiante había diligenciado en promedio tres veces el CME, para un total de 159 reportes.

\section{Análisis de datos}

Para el análisis de los datos se optó por un análisis de agrupamiento o clustering para la identificación de perfiles de compromiso. Se realizaron análisis segmentados para tres diferentes ventanas de tiempo, las cuales responden a cada una de las tres clases observadas. El uso de las ventanas de tiempo permitió capturar la variabilidad intra e inter individual en el tiempo. Para definir cada clúster se tomaron en cuenta las medidas de compromiso comportamental, cognitivo y afectivo para cada uno de los participantes. Todas las medidas fueron transformadas a puntajes $Z(M=0, D S=1)$. Para la clasificación en perfiles de compromiso, los puntajes por debajo de 0 (cero) fueron considerados bajos, los puntajes por encima de 1 (uno), altos, y los puntajes por encima de 0 (cero), pero por debajo de 1 (uno), moderados. Los análisis fueron llevados a cabo a través del software TANAGRA (1.4.50). Inicialmente se calculó el número óptimo de clústeres para cada ventana de tiempo a través de la técnica de agrupamiento jerárquico aglomerativa (HAC, por sus siglas en inglés). A continuación, se desarrolló un análisis de clústeres k-medias para cada ventana de tiempo, partiendo de las tres medidas normalizadas de compromiso. Esto permitió identificar los centroides de cada clúster y trazar perfiles de compromiso que representan a todos los sujetos miembros de dicho clúster. Además, se realizó un análisis descriptivo de las transiciones entre los clústeres entre sesiones. Esto permitió estimar el porcentaje de los sujetos que se movieron desde un perfil de compromiso particular en una sesión o si se mantuvieron con perfiles estables a lo largo del tiempo.

\section{Resultados}

Las siguientes tablas presentan las medias y desviaciones estándar para las diferentes dimensiones de compromiso en las diferentes sesiones. Como se puede observar en la Tabla 1, las medidas tendieron a ubicarse en puntajes medios para las tres dimensiones de compromiso. Los puntajes más altos fueron obtenidos en compromiso comportamental, seguido por afectivo y los puntajes más bajos fueron para el compromiso cognitivo. 
Tabla 1

Medias y desviaciones estándar de las dimensiones de compromiso para cada sesión.

\begin{tabular}{cccc}
\hline $\begin{array}{c}\text { Dimensión de } \\
\text { compromiso }\end{array}$ & $\begin{array}{c}\text { Sesión 1 } \\
\text { M (DE) }\end{array}$ & $\begin{array}{c}\text { Sesión 2 } \\
\text { M (DE) }\end{array}$ & $\begin{array}{c}\text { Sesión 3 } \\
\text { M (DE) }\end{array}$ \\
\hline Afectivo & $3.47(1.06)$ & $3.61(1.33)$ & $3.31(1.14)$ \\
Cognitivo & $3.17(1.14)$ & $3.31(1.53)$ & $3.00(1.18)$ \\
Comportamental & $3.94(1.0)$ & $3.75(.96)$ & $3.62(1.10)$ \\
\hline
\end{tabular}

Nota: $\mathrm{N}=53$ estudiantes, 159 respuestas.

Escala de calificación de compromiso: 1: nada, 2: un poco, 3: algo, 4: bastante, 5: mucho. Para esta muestra, puntaje mínimo: 1, puntaje máximo: 5 .

Las correlaciones son significativas entre compromiso afectivo y compromiso cognitivo en todas las sesiones. Igualmente, hay relación entre compromiso afectivo y comportamental en la sesión 1 y 3. Estas correlaciones no son altas, lo que indica que están relacionadas pero son diferentes. No hay correlaciones significativas entre compromiso cognitivo y comportamental en ninguna de las sesiones, lo cual resalta la diferencia de las dimensiones.

Tabla 2

Correlaciones entre las dimensiones de compromiso por sesión.

\begin{tabular}{|c|c|c|c|}
\hline & Afectivo & Cognitivo & Comportamental \\
\hline \multicolumn{4}{|l|}{ Sesión 1} \\
\hline Afectivo & - & $.416^{*}$ & $.464 *$ \\
\hline Cognitivo & $.416^{*}$ & - & .043 \\
\hline Comportamental & $.464 *$ & .043 & - \\
\hline \multicolumn{4}{|l|}{ Sesión 2} \\
\hline Afectivo & - & $.665^{*}$ & .080 \\
\hline Cognitivo & $.665^{*}$ & - & -.064 \\
\hline Comportamental & .080 & -.064 & - \\
\hline \multicolumn{4}{|l|}{ Sesión 3} \\
\hline Afectivo & - & $547 *$ & $.378 *$ \\
\hline Cognitivo & $547 *$ & - & .122 \\
\hline Comportamental & $.378 *$ & .122 & - \\
\hline
\end{tabular}

Nota: $\mathrm{N}=53$ estudiantes, 159 respuestas.

$* p<.001$

\section{Perfiles de compromiso en las clases de ciencias}

El primer objetivo de esta investigación era caracterizar los perfiles momentáneos de compromiso de los estudiantes en las clases de ciencias. Se encontraron cinco perfiles de compromiso: moderado, recreacional, universalmente bajo, renuente y racional. El compromiso moderado indica que todas las dimensiones de compromiso, cognitiva, afectiva y comportamental, puntuaron en un nivel medio (algo comprometidos); el recreacional indica niveles medios de compromiso comporta- 
mental y afectivo, y bajos niveles en compromiso cognitivo; el universalmente bajo implica que todas las dimensiones del compromiso están en un nivel bajo; el compromiso renuente implica que los estudiantes reportan un nivel de compromiso medio en la dimensión comportamental y bajo en las dimensiones cognitiva y afectiva; y finalmente, el compromiso racional indica que los estudiantes reportan un nivel medio en la dimensión cognitiva y bajo en la dimensión afectiva y comportamental.

La Figura 1 ilustra los perfiles de compromiso en las diferentes sesiones. En la primera sesión se encontraron tres tipos de perfiles: (1) compromiso moderado ( $\mathrm{n}=23$ estudiantes,
$43 \%$ ); (2) recreacional ( $\mathrm{n}=17$ estudiantes, correspondientes al $32 \%$ de la muestra); y (3) universalmente bajo $(\mathrm{n}=13$ estudiantes, $25 \%$ de los participantes). En la segunda sesión se presentaron los siguientes perfiles de compromiso: (1) renuente $(\mathrm{n}=23$ participantes, 44 $\%)$; (2) moderado $(\mathrm{n}=20$ estudiantes, $38 \%$ de los participantes); y (3) racional ( $\mathrm{n}=10$ estudiantes, correspondientes al $18 \%$ ). Por su parte, en la tercera sesión, los estudiantes mostraron los siguientes patrones de compromiso: (1) renuente $(\mathrm{n}=17$ participantes, igual a $32 \%$ de los estudiantes); (2) universalmente bajo $(\mathrm{n}=11$, correspondiente a $21 \%) ; \mathrm{y}(3)$ moderado $(n=25$, correspondiente a $47 \%)$.

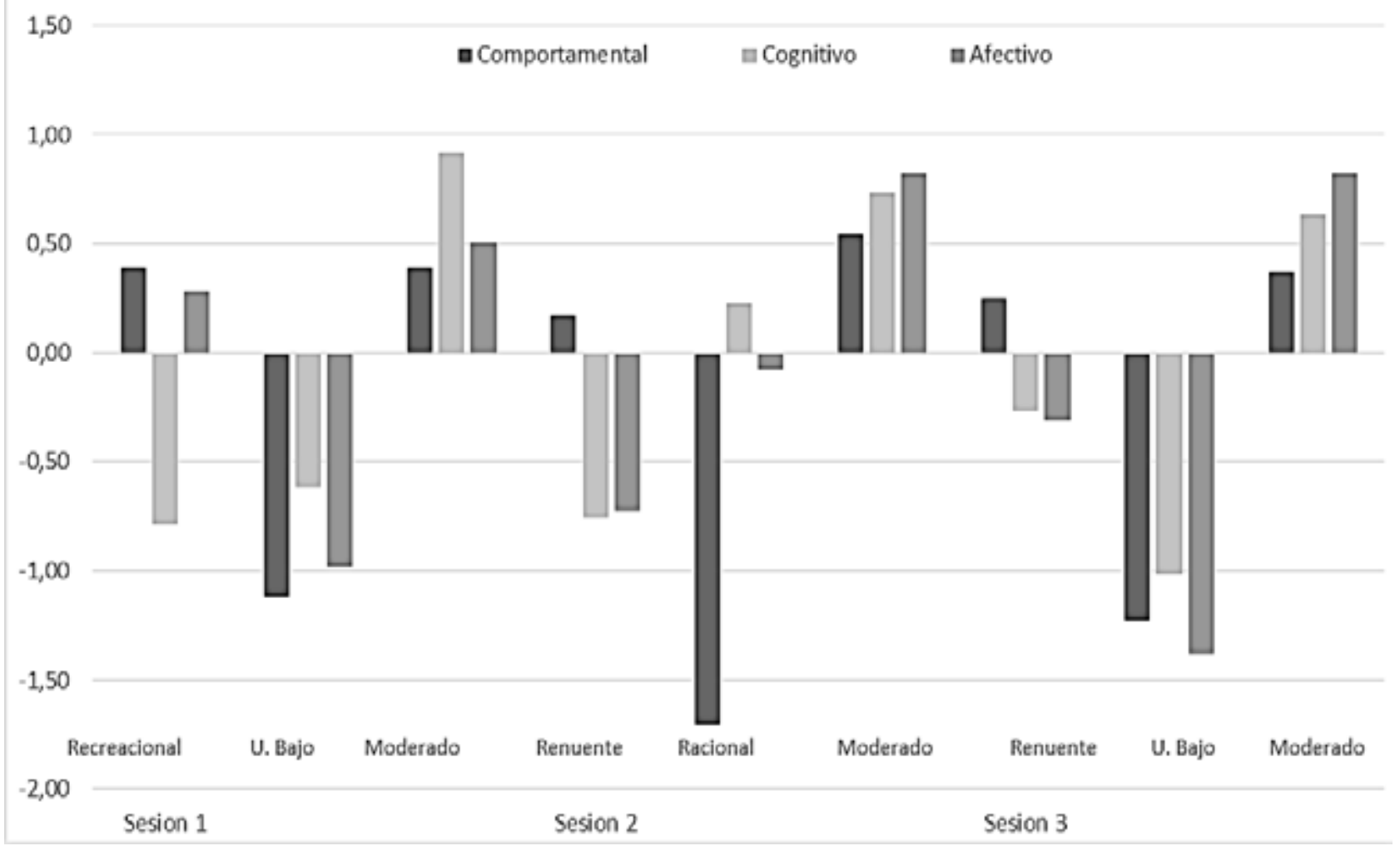

Figura 1. Perfiles de compromiso momentáneo a lo largo de las tres clases.

\section{Variación y permanencia de los patrones de compromiso a lo largo de la unidad temática}

El segundo objetivo de esta investigación indagaba si los perfiles de compromiso de los estudiantes variaban o se mantenían a lo largo de la unidad temática observada. En relación con la variabilidad de la sesión 1 a la sesión 2, el $23 \%$ de los estudiantes que mostraron compromiso moderado en la primera sesión conservó este patrón de compromiso, mientras 
que el $20 \%$ restante se dividió por igual en patrones de compromiso renuente y racional. Es decir, algunos perdieron la percepción de la importancia de la tarea y poder disfrutarla, pero continuaron esforzándose durante su realización, mientras que otros, aunque seguían percibiendo las actividades académicas como importantes, dejaron de disfrutarlas y de esforzarse durante su realización.

El $21 \%$ (del $32 \%$ ) de los estudiantes que mostraron un perfil recreacional en la sesión 1 en la sesión 2 evidenció un perfil renuente. Es decir, dejaron de disfrutar y mostrar interés en la tarea y pasaron solo a esforzarse por realizarla. Pero no todos estudiantes de este perfil siguieron este mismo patrón; el $8 \%$ pasó de recreacional a moderado en que disfrutaban de las actividades académicas durante la clase, entendían la importancia de las tareas abordadas para ellos mismos y para sus metas futuras y hacían el esfuerzo necesario para realizarlas. Por su parte, un $3 \%$ pasó de un compromiso recreacional a un compromiso racional, es decir, reconocen la importancia de las actividades de clase, aunque no hicieron su máximo esfuerzo durante su realización ni las disfrutaron.

Por su parte, de los estudiantes que mostraron un perfil universalmente bajo en la primera sesión ( $25 \%$ en total), el $13 \%$ pasó a renuente, a esforzarse por realizar la tarea y a concentrarse en ella aunque continuaban sin disfrutarla y sin entender su importancia; algunos pasaron a un compromiso moderado $(7 \%)$, mientas que un $5 \%$ cambió a un compromiso racional, es decir que reconocían la importancia de las tareas que estaban realizando, aunque no la disfrutaban ni ponían mucho esfuerzo y concentración en ellas. Para más detalles ver la Figura 2.

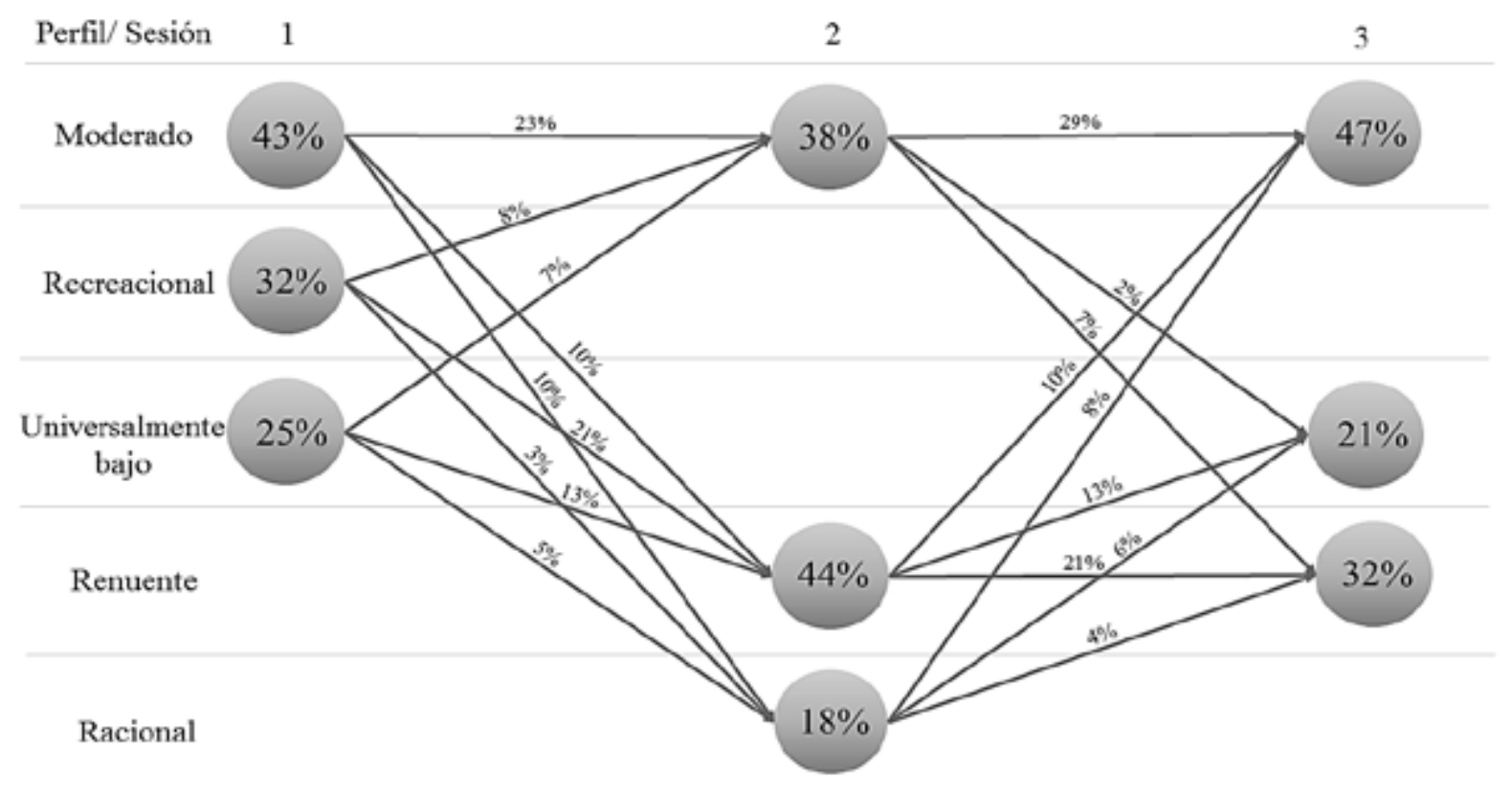

Figura 2. Variación y permanencia en los perfiles de compromiso a lo largo de tres sesiones.

En relación con la variabilidad de la sesión 2 a la sesión 3, del $44 \%$ de los participantes que presentaron un patrón de compromiso renuente, el $21 \%$ permaneció en este patrón; mientras que el $13 \%$ pasó a un compromiso universalmente bajo y el $10 \%$ reportó un compromiso moderado. Del $18 \%$ de los estudiantes que reportaron un compromiso racional, el $8 \%$ pasó a un compromiso moderado, mientras que el $6 \%$ pasó a un patrón de compromiso universalmente bajo y el $4 \%$ a un compromiso renuente. Por otra parte, del 
$38 \%$ de los estudiantes que habían reportado compromiso moderado, el $29 \%$ permaneció en este perfil, el $7 \%$ pasó a un compromiso renuente y el $2 \%$ pasó a un patrón de compromiso universalmente bajo.

En la figura también se observa que el perfil de compromiso moderado se mantuvo a lo largo de las tres sesiones con ligeras variaciones en el porcentaje de estudiantes que lo reportaban (un buen porcentaje de estudiantes mantienen el compromiso moderado a 10 largo de las 3 sesiones, $23 \%$ y $29 \%$ respectivamente). Igualmente, el perfil de compromiso renuente que se reporta en la sesión 2 se mantiene en la sesión 3 aunque decrece un poco. El perfil de compromiso universalmente bajo se presenta en las sesiones 1 y 3 , lo cual indica que fue el más variable pues no se sostiene a lo largo de las tres sesiones; sus integrantes, de la sesión 1 a la 2, se mueven hacia patrones de compromiso moderado, racional y renuente, y en la sesión 3 , sus integrantes provienen de los perfiles renuentes y racionales y solo un pequeño porcentaje de los ubicados en compromiso moderado.

\section{Discusión y conclusiones}

La primera pregunta de investigación indagó por los perfiles momentáneos de compromiso durante las clases de ciencias en estudiantes de secundaria de colegios colombianos. Los resultados obtenidos permiten confirmar la hipótesis 1 , pues muestran cinco perfiles de compromiso distribuidos a lo largo de tres sesiones observadas. Estos resultados permiten revalidar los perfiles propuestos por Conner y Pope (2013), que a su vez habían sido validados por Schmidt et al. (2017), quienes también reportan estos tipos de compromiso en estudiantes americanos. A diferencia de estos autores, en el presente estudio los estudiantes no reportaron perfiles de compromiso total, intencional, mentalizado y agradable.

Analizar el compromiso en términos de dimensiones y perfiles es importante porque dicho análisis muestra la multidimensiona- lidad y dinamismo del constructo (Christenson et al., 2012; Eccles, 2016). En relación con la multidimensionalidad, este estudio aporta evidencia al grupo de investigaciones y estados de la cuestión que toman en cuenta las tres dimensiones del compromiso para describir la complejidad del fenómeno $\mathrm{y}$ explicar cómo las posibles combinaciones entre ellas forman patrones que describen las diferentes maneras en que los estudiantes abordan la vida académica (Conner y Pope, 2013; Schmidt et al., 2017).

El compromiso moderado fue el perfil más frecuente en este estudio y el compromiso total no se presentó. Estos resultados coinciden parcialmente con los de Conner y Pope (2013), Osborne, Simon y Collins (2003) y Schmidt et al. (2017), quienes reportaron mayor frecuencia de perfiles de compromiso bajo en los estudiantes de ciencias y poca frecuencia de perfiles de compromiso total. Por otro lado, en los patrones de compromiso bajo (sesiones 1 y 3 ) los puntajes de las dimensiones fueron marcadamente bajos en comparación con los otros perfiles, lo que indica que los estudiantes están en un estado de apatía, no ven valor en lo que aprenden, no les interesan las tareas y ponen muy poco esfuerzo en su realización.

La segunda pregunta de investigación de este estudio indagaba por la variabilidad o permanencia de los perfiles a lo largo de unidades temáticas de tres sesiones cada una. Mostrar cómo cambian o se mantienen los perfiles a lo largo del tiempo aporta evidencia respecto del carácter emergente y variable del compromiso, y pone en cuestionamiento aproximaciones que lo presentan como un fenómeno estático o como un rasgo particular de los estudiantes (Csikszentmihalyi et al., 2005; Schmidt et al., 2017; Strati et al., 2017).

La variabilidad de los perfiles de compromiso a lo largo de las tres sesiones permite confirmar la hipótesis 2. La primera sesión se caracteriza porque los estudiantes en la clase presentan un perfil moderado; conviven con otro grupo de estudiantes que presentan un 
perfil recreacional, y con el grupo que presenta perfil universalmente bajo. En la sesión 2 emergen dos nuevos perfiles de compromiso (renuente y racional) y se mantiene el moderado, mientras que desaparece el universalmente bajo. En la sesión 3 se mantiene el compromiso moderado y el renuente, y reaparece el compromiso universalmente bajo.

Al revisar los perfiles por sesión, se encuentra que los grupos que componen dichos perfiles no están conformados por los mismos estudiantes. Esto marca no solo una variabilidad en los perfiles entre sesiones, sino que a la vez permite describir las oscilaciones y trayectorias intraindividuales de compromiso. Esto implica que un estudiante no necesariamente presenta el mismo patrón de compromiso a lo largo de las tres sesiones de clase $y$, aunque algunos estudiantes tienden a tener perfiles estables a lo largo del tiempo, otros muestran una mayor variabilidad.

La descripción de los perfiles de compromiso y su correspondiente análisis a lo largo de la unidad temática proponen preguntas y retos diferenciados para los estudiosos de las interacciones entre docentes y estudiantes, y su relación con el compromiso. A continuación, se presentan las preguntas que surgen del análisis de los perfiles de compromiso, las posibles respuestas que se pueden plantear a partir de la literatura sobre el tema, y los retos para futuras investigaciones y para el apoyo docente que debería enfocarse en atender, de manera diferenciada, a los estudiantes de acuerdo a las necesidades asociadas a los perfiles emergentes en las interacciones educativas.

En la sesión 1 se evidenciaron los siguientes perfiles, presentados de mayor a menor porcentaje: moderado, recreacional y universalmente bajo. En relación con los estudiantes que presentan un compromiso moderado, ¿cómo lograr que los estudiantes pasen a un compromiso total? Este grupo requiere tareas que le susciten un mayor interés y disfrute, lo que podría influir en su compromiso comportamental y llevarlos a hacer mayores esfuerzos en tareas que les interesen y cuya realización les genere disfrute. En este sentido la clave para este grupo podría surgir de los estudios sobre cómo promover el interés individual (Hidi y Renninger, 2006). Según los autores, un punto importante es tomar en cuenta las necesidades e intereses personales de los estudiantes y relacionarlos con los contenidos $\mathrm{y}$ actividades académicas $\mathrm{y}$, con base en esto, guiar las actividades de clase, seleccionar los materiales a utilizar y proponer evaluaciones. Estos aspectos están relacionados con la instrucción diferenciada (Akos, Cockman y Strickland, 2007). Como se ve en las siguientes sesiones, en este estudio el paso del compromiso moderado al total no se logra. Los resultados muestran que la gran mayoría de los estudiantes que evidenciaron este perfil en la sesión 1 lo mantienen en las sesiones 2 y 3, mientras que algunos terminan la unidad temática haciendo esfuerzos por concentrarse, pero sin experimentar disfrute ni interés, y otros pocos, un perfil de bajo compromiso. Esto último indicaría que, para los participantes de este estudio no se pasa directamente de un compromiso moderado a un compromiso total; por el contrario, el compromiso puede decaer totalmente $\mathrm{y}$, en este sentido, se torna más urgente tomar en cuenta estos intereses personales de los estudiantes para los procesos educativos.

El segundo perfil más frecuente fue el recreacional; en relación con este perfil surge la pregunta: ¿qué prácticas pedagógicas usar para que los estudiantes encuentren relevancia personal en las temáticas de la clase? Los resultados de investigaciones sobre el sentido personal y social de lo que se aprende podrían guiar el desarrollo de prácticas educativas pertinentes para estos estudiantes (Assor, Kaplan y Roth, 2002; Eccles, 2005; Shumow y Schmidt, 2014). Estos autores señalan que los estudiantes otorgan mayor valor a aquellas tareas que son acordes a su identidad y valores personales. En este sentido, los docentes deben hacer un esfuerzo por promover una relación empática con sus estudiantes y comprender sus mundos, es decir sus objetivos, intereses 
y necesidades para luego vincularlos con las tareas escolares (Assor et al., 2002; Laudadío y Mazzitelli, 2018). Esta percepción de relevancia no parece darse para algunos estudiantes de este perfil en el transcurso de las clases. En la sesión 2, un gran porcentaje de estudiantes que presentaron este perfil pasa a un perfil renuente, es decir que no solo siguen sin ver la importancia de lo que aprenden, sino que dejan de disfrutar la clase y mantienen solamente algo de esfuerzo y concentración. De la sesión 2 a la 3, algunos permanecen renuentes, pero otra buena parte baja totalmente su compromiso. Un buen porcentaje de estudiantes presentó un compromiso universalmente bajo en la sesión 1. Ante este perfil surge la pregunta: ¿qué características deben tener las tareas, los materiales, las interacciones para comprometer a los estudiantes que no encuentran relevantes ni disfrutan las actividades escolares y no se concentran ni se esfuerzan durante su realización? "Apatía" es el concepto que puede resumir las características de este grupo de estudiantes. La literatura sobre la apatía reporta que esta es una experiencia emocional muy negativa dado que implica poca concentración, muy poco disfrute, poca o ninguna percepción de relevancia personal de la tarea, y sus efectos para el aprendizaje, la creatividad y el desempeño son desastrosos (Ochoa-Angrino, 2012; Massimini y Carli, 1998; Nakamura y Csikszentmihalyi, 2002). La teoría de la motivación emergente (Nakamura y Csikszentmihalyi, 2002) y los estudios sobre promoción de la autonomía (Assor, et al., 2002) sugieren que, en estos casos, los profesores deben seleccionar las actividades académicas partiendo de la motivación intrínseca de los estudiantes, de sus intereses, en lugar de centrarse en sus deficiencias. Investigaciones recientes de Turner, Christensen, Kackar-Cam, Trucano y Fulmer (2014) apoyan la relación entre el fomento de la autonomía y el mejoramiento en el compromiso de los estudiantes. En este estudio, la gran mayoría de los estudiantes de este perfil se movieron hacia un perfil renuente en la segunda sesión, mientras otros lo hicieron hacia moderado y unos pocos, a racional. Los estudiantes empiezan a esforzarse y concentrarse $\mathrm{o}$ a experimentar moderados niveles de compromiso comportamental, cognitivo y afectivo, y unos pocos pudieron encontrar algo de sentido en las clases. Parece que predominó la tendencia a realizar esfuerzos en lugar de percibir importancia, interés y disfrute, lo que indicaría que las prácticas educativas podrían no estar tomando en cuenta la motivación intrínseca de los estudiantes.

En la segunda sesión se presentaron los perfiles renuente, moderado y racional. En relación con el perfil renuente: ¿cómo deberían los docentes abordar a los estudiantes que se están esforzando por cumplir con una tarea que no encuentran importante y en la que no encuentran disfrute? En consideración a que estos estudiantes parecen estar guiados por la motivación extrínseca (calificaciones, complacer a los profesores y a los padres), los estudios sobre promoción de la autonomía podrían guiar las acciones docentes (Reeve y Cheon, 2016; Ryan y Deci, 2000; Su y Reeve, 2011). El apoyo a la autonomía se caracteriza por crear escenarios educativos que, partiendo de los intereses, las necesidades y los puntos de vista de los estudiantes, les permitan resolver los problemas a su propio ritmo, les proporcionen alternativas para escoger las temáticas, los materiales, las maneras de resolver las tareas, las estrategias para abordarlos y, en la medida de lo posible, usar lenguaje y gestos poco controladores. Igualmente, permitir y aceptar la expresión de los afectos negativos (Su y Reeve, 2011). Cuando por alguna razón los niños deban realizar tareas que no disfrutan deben ofrecerse explicaciones y justificaciones personales y sociales sobre la importancia de la tarea (Assor et al., 2002; Ryan y Deci, 2000). Aproximadamente la mitad de los estudiantes muestra este mismo perfil en la sesión 3, mientras el resto se mueve, en partes más o menos iguales, hacia a un perfil moderado o bajo. Estos resultados parecen indicar que una buena parte de los estudiantes 
hace su mejor esfuerzo por concentrarse en las tareas académicas, aunque no las encuentren muy significativas ni las disfruten. En relación con el perfil moderado, en la segunda sesión se observan varios aspectos: el grupo de estudiantes que reporta este perfil disminuye con respecto al de la sesión 1 , aunque un poco más de la mitad del grupo original se mantiene; por otra parte, se observa que los perfiles recreacional y universalmente bajo contribuyen a engrosar el grupo de perfil moderado en la sesión 2. En el caso de los estudiantes del perfil recreacional, pasaron de no encontrar sentido a la clase en la primera sesión, a hacerlo en la segunda. Con menor frecuencia está el grupo con perfil racional. Ante este perfil la pregunta que surge es ¿cómo abordar al grupo de estudiantes que ve algo de valor personal en las tareas que realiza, pero en las que el disfrute es mínimo y el esfuerzo y la concentración son casi nulos? Algunas pistas clave para orientar el trabajo de estos estudiantes las podrían ofrecer estudios sobre el interés situacional. Palmer (2009) y Cooper (2014) señalan que el uso de actividades prácticas, físicas y de involucramiento social (juegos), la novedad, la sorpresa y el trabajo en grupo son estrategias que han mostrado efectividad en disparar y mantener el interés situacional de los estudiantes en clases de ciencias y podrían tener un rol compensatorio cuando los estudiantes se sienten poco conectados con la clase. Hagay y Baram-Tsabari (2015) reportan que la búsqueda de información sobre preguntas autoformuladas también promueve y mantiene el interés. Los datos de esta investigación reportan que un poco más de la tercera parte de estudiantes con este perfil pasan en la sesión 3 a un compromiso moderado, pero otra tercera parte del grupo deja de percibir importancia en la tarea y disminuye totalmente su compromiso. Esto es preocupante porque señala que durante la clase no se aprovecha la importancia incipiente que los estudiantes encuentran en la clase y su compromiso puede decaer totalmente.

Finalmente, en la tercera sesión se presentan los perfiles moderado, renuente y universalmente bajo. Casi la mitad de los estudiantes se perciben concentrados, hacen esfuerzos por comprender, encuentran importancia en los cursos, están algo interesados y los disfrutan. Buena parte de esos estudiantes han permanecido comprometidos a lo largo de las diferentes sesiones, mientras otros en la sesión anterior habían reportado un compromiso renuente, lo que indica que mejoró la percepción de importancia, el interés y el disfrute, y otros terminaron haciendo un mayor esfuerzo que al comienzo de la sesión. En este último grupo, podría pensarse que la cercanía de evaluación de la unidad temática puede haber influido en la variación en el grado de esfuerzo y concentración. En relación con el grupo de renuentes, terminan la unidad temática esforzándose sin percibir relevancia ni interés y disfrute. Del grupo de estudiantes que reporta un perfil de compromiso bajo, la gran mayoría proviene del perfil renuente seguido del racional. Esto podría indicar que el poco esfuerzo que hacían decayó al no percibirse importancia, interés ni disfrute, y que los que encontraban algo de sentido (racional) dejaron de percibirlo al final del curso.

En consonancia con la literatura revisada, el presente estudio concuerda con que es necesaria una instrucción diferenciada que responda a las necesidades de los estudiantes de acuerdo a cada perfil. No obstante, es también necesario considerar la dinámica de los perfiles y su variabilidad intraindividual, lo cual implica que el docente debe hacer una lectura de lo que ocurre en el momento de las interacciones docentes, pues el perfil de compromiso del estudiante puede variar, no solo de clase a clase, sino dentro de una misma clase. Además, esta variación puede ser más o menos amplia de acuerdo al estudiante que se esté analizando. En esta medida, unos estudiantes requerirán apoyo para mantenerse estables en perfiles de compromiso deseables (compromiso alto o moderado) mientras otros necesitan interacciones que los movilicen hacia perfiles más deseables. 
A manera de conclusión, se identificaron cinco perfiles momentáneos de compromiso cognitivo, afectivo y comportamental de estudiantes de secundaria durante clases de ciencias. Los perfiles mostraron permanencia y variación a lo largo de tres sesiones. El perfil más frecuente y permanente fue el compromiso moderado, que se presentó a lo largo de las tres sesiones, mientras que el compromiso universalmente bajo se presentó en la primera y la última sesión; el perfil renuente se presentó en la segunda y tercera sesión. Los diferentes perfiles momentáneos suscitan diferentes preguntas y retos educativos para los docentes. Se discute la relación entre los tipos de compromiso y las características de las prácticas educativas. Se resalta la importancia de usar prácticas que promuevan el interés, la autonomía y la relevancia. Finalmente, se sugiere instrucción diferenciada no solo según el tipo de perfiles, sino según la variación momentánea de los estudiantes en dichos perfiles.

\section{Limitaciones del estudio}

Aunque los resultados del estudio revalidan los perfiles de compromiso propuestos por Conner y Pope (2013), validados por Schmidt et al. (2017), la generalidad de los resultados es limitada, pues la muestra de este estudio es muy pequeña comparada con las de los estudios en los que se basa. Una futura línea de investigación deberá contrastar los resultados de esta investigación con los resultados de investigaciones con muestras más representativas de estudiantes de secundaria colombianos. Una segunda limitación está relacionada con la poca densidad de datos (tres medidas por participante). Este número de medidas, aunque recoge cambios en el tiempo a lo largo de una unidad temática, es limitado para capturar la variabilidad del compromiso a largo de una clase y, por supuesto, de periodos más extensos de tiempo, por ejemplo, la variabilidad en un curso. Finalmente, en este estudio no se analizan factores de la rela- ción profesor-estudiante que puedan estar relacionados con los perfiles de compromiso descritos y con su variabilidad o permanencia.

Próximos estudios deberían combinar los tres elementos: la multidimensionalidad del compromiso, los perfiles momentáneos y la relación con las características de las situaciones educativas. Schmidt et al. (2017) señalan que de esta forma los profesores no solo conocen las diferentes maneras en que los estudiantes se comprometen durante las clases, sino también las condiciones que favorecen la emergencia de ciertos patrones de compromiso.

\section{Referencias bibliográficas}

Akos, P., Cockman, C. y Strickland, C. (2007). Differentiating classroom guidance. Professional School Counseling, 10, 455-463. http:// doi.org/10.1177/2156759X0701000502

Assor, A., Kaplan, H. y Roth, G. (2002). Choice is good, but relevance is excellent: Autonomy enhancing and suppressing teacher behaviors predicting students' engagement in schoolwork. British Journal of Educational Psychology, 72(2), 261-278. http://doi. org/10.1348/000709902158883

Chang, D. F., Chien, W. C. y Chou, W. (2016). Meta-analysis approach to detect the effect of student engagement on academic achievement. ICIC Express Letters, 10(10), 2441-2446. http://doi.org/10.3102/0034654311421793

Christenson, S. L., Reschly, A. L. y Wiley, C. A. (2012). Handbook of Student Engagement. New York, NY : Springer Science.

Conner, J. O. y Pope, D. C. (2013). Not Just Robo-Students: Why Full Engagement Matters and How Schools Can Promote It. Journal of Youth and Adolescence, 42(9), 1426-1442. http://doi.org/10.1007/s10964-013-9948-y

Cooper, K. S. (2014). Eliciting Engagement in the High School Classroom: A Mixed-Methods Examination of Teaching Practices. American Educational Research Journal, 51(2), 636-402. http://doi.org/10.3102/0002831213507973

Csikszentmihalyi, M. y Larson, R. (1987). Vali- 
dity and Reliability of the Experience-Sampling Method. Journal of Nervous $y$ Mental Disease, 175(9), 526-36. http://doi. org/10.1097/00005053-198709000-00004

Csikszentmihalyi, M., Abuhamdeh, S. y Nakamura, J. (2005). Flow. En A. Elliot y C. Dweck (Eds.), Handbook of competence and motivation (pp. 598-608). New York: Guilford.

De Laet, S., Colpin, H., Vervoort, E., Doumen, S., Van Leeuwen, K., Goossens, L. y Verschueren, K. (2015). Developmental trajectories of children's behavioral engagement in late elementary school: Both teachers and peers matter. Developmental Psychology, 51, 1292-1306. http://doi.org/10.1037/a0039478

Eccles, J. S. (2005). Subjective task value and the Eccles et al. model of achievement-related choices. En A. Elliot y C. Dweck (Eds.), Handbook of competence and motivation (pp. 105-121). New York: The Gilford Press.

Eccles, J.S. (2016). Engagement: Where to next? Learning and Instruction, 43, 71-75. http://doi. org/10.1016/j.learninstruc.2016.02.003

Furrer, C. J., Skinner, E. A. y Pitzer, J. R. (2014). The Influence of Teacher and Peer Relationships on Students' Classroom Engagement and Everyday Motivational Resilience. Yearbook of the National Society for the Study of Education, 113(13), 101-123.

Gillet, N., Morin, A. J. S. y Reeve, J. (2017). Stability, change, and implications of students' motivation profiles: A latent transition analysis. Contemporary Educational Psychology, 1-52. http://doi.org/10.1016/j. cedpsych.2017.08.006.

Hagay, G. y Baram-Tsabari, A. (2015). A strategy for incorporating students' interests into the high school science classroom. Journal of Research in Science Teaching, 57(7), 949-978. https://doi.org/10.1002/tea.21228.

Hektner, J. M., Schmidt, J. A. y Csikszentmihalyi, M. (2007). Experience sampling method: Measuring the quality of everyday life. Thousand Oaks, CA: Sage Publishing.

Hernández, R. y Carranza, R. (2017). Felicidad, optimismo y autorrealización en estudiantes de un programa de educación superior para adultos. Interdisciplinaria, 34(2), 307-325. http://doi.org/10.16888/interd.2017.34.2.5

Hidi, S. y Renninger, K. A. (2006). The four phase model of interest development. Educational Psychologist 41(2), 111-127. http://doi. org/10.1207/s15326985ep4102_4

Jang, H., Kim, E. J. y Reeve, J. (2016). Why students become more engaged or more disengaged during the semester: A self-determination theory dual-process model. Learning and Instruction, 43, 27-38. http://doi.org/10.1016/j. learninstruc.2016.01.002

Ketonen, E., Haarala-Muhonen, A., Hirsto, L., Hänninen, J., Wähälä, K. y Lonka, K. (2016). Am $\mathrm{I}$ in the right place? Academic engagement and study success during the first year at university. Learning and Individual Differences. 51, 141-148. http://doi.org/10.1016/j. lindif.2016.08.017

Lam, S., Jimerson, S., Shin, H., Cefai, C., Veiga, F. H., Hatzichristou, C...Zollneritsch, J. (2016). Cultural universality and specificity of student engagement in school: The results of an international study from 12 countries. British Journal of Educational Psychology, 86(1), 137-153. https://doi.org/10.1111/bjep.12079

Lawson, M. y Masyn, K. (2015). Analyzing Profiles and Predictors of Students' Social-Ecological. Journal of School Psychology, 53. 63-86. http://doi.org/10.1016/j.jsp.2014.11.004

Laudadío, J. y Mazzitelli, C. (2018). Adaptación y validación del Cuestionario de Relación Docente en el Nivel Superior. Interdisciplinaria, 35(1), 153-170. http://doi.org/10.16888/ interd.2018.35.1.8

Lei, H., Chui, Y. y Zhou, W. (2018). Relationships between student engagement and academic achievement: A meta-analysis. Social Behavior and Personality, 46(3), 516-528. https:// doi.org/10.2224/sbp.7054

Martin, A. J., Papworth, B., Ginns, P., Malmberg, L. E., Collie, R. y Calvo, R. A. (2015). Real-time motivation and engagement during a month at school: Every moment of every day for every student matters. Learning and Individual Differences, 38, 26-35. http://doi. org/10.1016/j.lindif.2015.01.014 
Massimini, F. y Carli, M. (1988). The systematic assessment of flow in daily experience. En M. Csikszentmihalyi e I.S. Csikszentmihalyi (Eds.), Optimal experience: Psychological studies of flow in consciousness (pp. 266-287). New York: Cambridge University Press.

Ministerio de Educación Nacional (2017). Colombia en PISA 2015. Informe nacional de resultados. Bogotá. Recuperado de http:// www2.icfes.gov.co/en/docman/instituciones-educativas-y-secretarias/evaluaciones-internacionales-investigadores/pisa/ pisa-2015/2934-informe-nacional-pisa-2015/ file? force-download $=1$.

Molenaar, P. (2004). A manifest on psychology as idiographic science: bringing the person back into scientific psychology, this time forever, Measurement, 2(4), 201-218. http://doi.org/10.1207/ s15366359mea0204_1

Nakamura, J. y Csikszentmihalyi, M. (2002). The concept of flow. En C.R. Snyder y S.J. López (Eds.), Handbook of positive psychology (pp. 89-105). New York: Oxford University Press.

Ochoa-Angrino, S. (2012). Factors related to students' engaged reading in high school science classrooms (Tesis doctoral). Northern Illinois University, EE.UU. Recuperado de http:/gradwor ks.umi. com/35/13/3513148.html

Ochoa-Angrino, S., Montes-González, J. A. y Rojas-Ospina, T. (2018). Percepción de habilidad, reto y relevancia como predictores de compromiso cognitivo y afectivo en estudiantes de secundaria. Universitas Psychologica, 17(5), 1-18. http://doi. org/10.11144/Javeriana.upsy17-5.phrr

Osborne, J., Simon, S., y Collins, S. (2003). Attitudes towards science: A review of the literature and its implications. International Journal of Science Education, 25, 1049- 1079. https://doi. org/10.1080/0950069032000032199

Palmer, D. H. (2009). Student interest generated during an inquiry skill lesson. Journal of Research in Science Teaching, 46(2),
147-165. http://doi.org/10.1002/tea.20263.

Reeve, J. y Cheon, S. H. (2016). Teachers become more autonomy supportive after they believe it is easy to do. Psychology of Sport and Exercise, 22, 178-189. http:// doi.org/10.1016/j. psychsport.2015.08.001

Rigo, D. (2017). Docentes, tareas y alumnos en la definición del compromiso: Investigando el aula de nivel primario de educación. Educação em Revista, 33, 1-24. http:// doi.org/10.1590/0102-4698154275

Ryan, R. M. y Deci, E. L. (2000). Self-Determination Theory and the Facilitation of Intrinsic Motivation, Social Development, and Well-Being. American Psychological Association, 55(1), 68-78. http://doi. org/10.1037110003-066X.55.1.68

Salmela-Aro, K., Moeller, J., Schneider, B. y Lavonen, J. (2016). Integrating the light and dark sides of student engagement using person-oriented and situation-specific approaches, Learning and Instruction, 43, 61-70. http://doi.org/10.1016/j.learninstruc.2016.01.001

Salvatore, S. y Valsiner, J. (2010). Between the general and the unique: Overcoming the nomothetic versus idiographic opposition. Theory y Psychology, 20(6), 817-833. https://doi. org/10.1177/0959354310381156

Schmidt, J. A. y Smith, M. C. (2008). Looking inside high school science classrooms: An exploration of males 'and females' subjective experience. National Science Foundation, Human Resources Directorate, program on Gender in Science and Engineering. Grant No. HRD-0827526.

Schmidt, J. A., Rosenberg, J. M. y Beymer, P. N. (2017). A person-in-context approach to student engagement in science: Examining learning activities and choice. Journal of Research in Science Teaching, 55(1), 19-43. http://doi.org/10.1002/tea.21409

Shernoff, D. J. y Bempechat, J. (Eds.). (2014). Engagingyouth in schools: Evidence-based models to guide future innovations. New York: NSSE Yearbook, Teachers College 
Record.

Shernoff, D. J., Ruzek, E. A. y Sinha, S. (2016). The influence of the high school classroom environment on learning as mediated by student engagement. School Psychology International, 38(2), 201-218. http://doi.org/10.1177/0143034316666413

Shumow, L. y Schmidt, J. A. (2014). Enhancing Adolescents' Motivation for Science. USA: Corwin Press.

Siegler, R. (1994). Cognitive Variability: A Key to Understanding Cognitive Development. Current Directions in Psychological Science, 3(1), 1-5. https://doi. org/10.1111/1467-8721.ep10769817.

Strati, A. D., Schmidt, J. A. y Maier, K. S. (2017). Perceived challenge, teacher support, and teacher obstruction as predictors of student engagement. Journal of Educational Psychology, 109(1), 131. http://doi.org/10.1037/edu0000108

$\mathrm{Su}$, Y. L. y Reeve, J. (2011). A meta-analysis of the effectiveness of intervention programs designed to support autonomy. Educational Psychology Review, 23(1), 159-188. http://doi.org/10.1007/s10648-010-9142-7

Turner, J. C., Christensen, A., Kackar-Cam, H. Z., Trucano, M. y Fulmer, S. M. (2014). Enhancing students' engagement: Report of a 3-year intervention with middle school teachers. American Educational Research Journal, 51, 1195-1226. http:// doi.org/10.3102/0002831214532515
Van Geert, P. y Van Dijk, M. (2002). Focus on variability: New tools to study intra-individual variability in developmental data. Infant Behavior y Development, 25, 340-374. http://doi.org/10.1016/S01636383 (02)00140-6

Wang, M. y Holcombe, R. (2010). Adolescents' perceptions of school environment, engagement, and academic achievement in middle school. American Educational Research Journal, 47, 633-62. http://doi. org/10.3102/000283120936120

Wang, M. y Eccles, J. S. (2013). School context, achievement motivation, and academic engagement: A longitudinal study of school engagement using a multidimensional perspective. Learning and Instruction, 28, 12-23. https://doi.org/10.1016/j. learninstruc.2013.04.002

Watt, H. M. G., Carmichael, C. y Callingham, R. (2017). Students' engagement profiles in mathematics according to learning environment dimensions: Developing an evidence base for best practice in mathematics education. School Psychology International, 38(2), 166-183. http://doi. org/10.1177/0143034316688373.

Zuzanek, J. (1999, May). Experience Sampling Method: Current and potential research applications. Paper presented at the Workshop on Time-Use Measurement and Research, National Research Council, Washington, DC.

Recibido: 9 de septiembre de 2018 Aceptado: 24 de julio de 2020 